\title{
A low fraction of nitrogen in molecular form in a dark cloud
}

\author{
S. Maret ${ }^{1}$, E. A. Bergin ${ }^{1} \&$ C. J. Lada ${ }^{2}$
}

Nitrogen is the fifth most abundant element in the Universe. In the interstellar medium, it has been thought to be mostly molecular $\left(\mathrm{N}_{2}\right)^{1}$. However, $\mathrm{N}_{2}$ has no observable rotational or vibrational transitions, so its abundance in the interstellar medium remains poorly known. In comets, the $\mathrm{N}_{2}$ abundance is very low ${ }^{2,3}$, while the elemental nitrogen abundance is deficient with respect to the solar value. Moreover, large nitrogen isotopic anomalies are observed in meteorites and interstellar dust particles ${ }^{4}$. Here we report the $\mathrm{N}_{2} \mathrm{H}^{+}$(and by inference the $\mathrm{N}_{2}$ ) abundance inside a cold dark molecular cloud. We find that only a small fraction of nitrogen in the gas phase is molecular, with most of it being atomic. Because the compositions of comets probably reflect those of dark clouds ${ }^{5}$, this result explains the low $\mathrm{N}_{2}$ abundance in comets. We argue that the elemental nitrogen abundance deficiency in comets can be understood if the atomic oxygen abundance is lower than predicted by present chemical models. Furthermore, the lack of molecular nitrogen in molecular clouds explains the nitrogen anomalies in meteorites and interstellar dust particles, as nitrogen fractionation is enhanced if gaseous nitrogen is atomic ${ }^{6}$.

For more than three decades, chemical models ${ }^{1}$ have predicted that nitrogen is mostly in the form of $\mathrm{N}_{2}$ in dense interstellar clouds. Observations of $\mathrm{N}_{2} \mathrm{H}^{+}-$a chemical daughter product of $\mathrm{N}_{2}$-have been used, together with $\mathrm{HCO}^{+}$observations, to estimate the $\mathrm{N}_{2} / \mathrm{CO}$ abundance ratio in molecular clouds ${ }^{7}$. In these clouds, both ions are formed from the reaction with the $\mathrm{H}_{3}^{+}$ion with either $\mathrm{N}_{2}$ or $\mathrm{CO}$, and are destroyed mostly by recombination with electrons. Both reactions occur at a similar rate for the two ions, and so the measure of the $\mathrm{HCO}^{+} / \mathrm{N}_{2} \mathrm{H}^{+}$abundance ratio is a direct measure of the $\mathrm{N}_{2} / \mathrm{CO}$ ratio, and in turn of the $\mathrm{N}_{2}$ abundance if the $\mathrm{CO}$ abundance is known. Observations of this ratio indicate that most nitrogen is in the form of $\mathrm{N}_{2}$ in molecular clouds, thereby supporting theoretical predictions.

This approach is too simple, omitting consideration of the many other formation and destruction routes of $\mathrm{N}_{2} \mathrm{H}^{+}$that are known to exist in interstellar clouds. For example, $\mathrm{N}_{2} \mathrm{H}^{+}$is destroyed via a reaction with $\mathrm{CO}$ that has no counterpart for $\mathrm{HCO}^{+}$. Therefore, to derive the $\mathrm{N}_{2}$ abundance from $\mathrm{N}_{2} \mathrm{H}^{+}$, one must consider an expanded network of gas-phase chemical reactions. Furthermore, in dense and cold parts of molecular clouds, molecules can condense on interstellar grains and form an icy mantle around them, a phenomenon known as depletion. Depletion of several molecules is observed in numerous cold molecular cores ${ }^{8}$. For example, CO and CS abundances are estimated to decrease by several orders of magnitude near the core centres, where the density is highest ${ }^{9}$. Although $\mathrm{N}_{2} \mathrm{H}^{+}$itself does not deplete on grain mantles, its abundance also seems to be reduced in the centre regions of starless cores with respect to the core edges ${ }^{9,10}$.

This disappearance from the gas phase is probably due to the freeze-out of $\mathrm{N}_{2} \mathrm{H}^{+}$parent molecule, $\mathrm{N}_{2}$, onto the grain surfaces. The ability of a molecule or atom to remain on a grain once it has frozen out depends on its binding energy to the grain surface. Observations of $\mathrm{C}^{18} \mathrm{O}$ and $\mathrm{N}_{2} \mathrm{H}^{+}$emission are well reproduced by gas-phase chemistry models including depletion, provided that the ratio between the binding energies of $\mathrm{N}_{2}$ and $\mathrm{CO}$ is between 0.5 and 0.75 (ref. 9). However, recent laboratory measurements indicate that

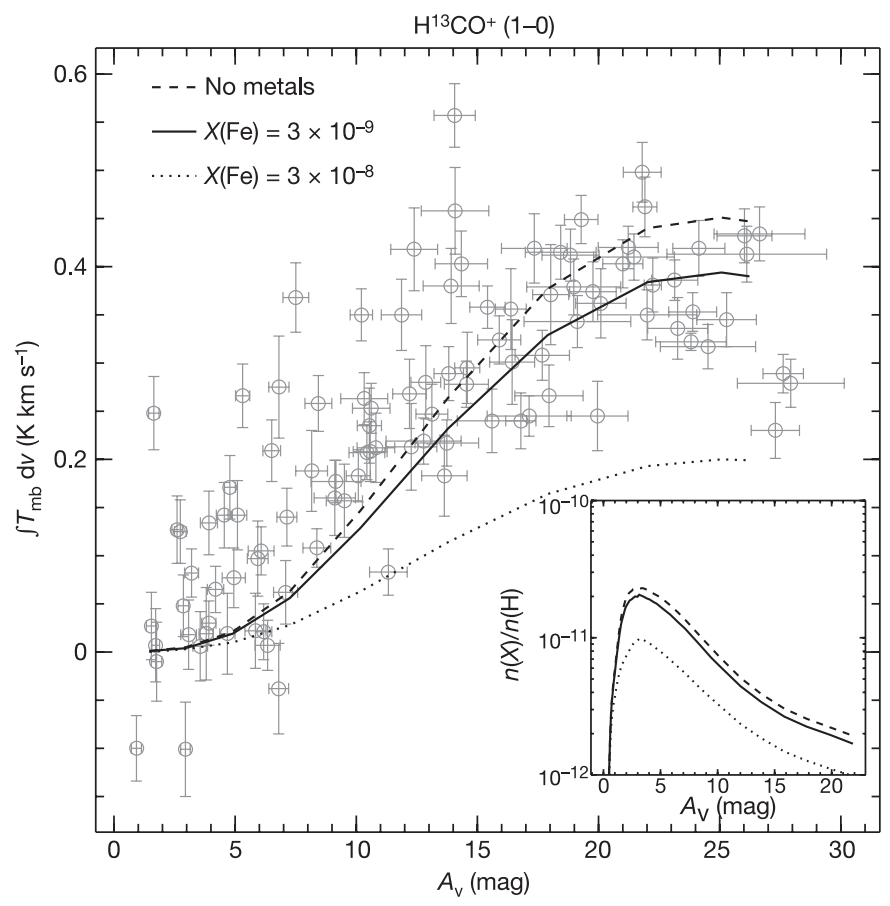

Figure 1 Determination of the metal abundance inside the B68 core. Grey points with error bars $(1 \sigma)$ represent the observed $\mathrm{H}^{13} \mathrm{CO}^{+}(1-0)$ line intensities in main beam temperature units $\left(T_{\mathrm{mb}}\right)$ as a function of the visual extinction in the core $\left(A_{\mathrm{v}}\right)$. The black lines represent the model predictions for different metal abundances. The inset shows the corresponding $\mathrm{H}^{13} \mathrm{CO}^{+}$ abundances $(X)$. The $\mathrm{H}^{13} \mathrm{CO}^{+}$abundances have been computed as a function of the visual extinction by a chemical network that includes depletion and desorption (the removal of molecules from grain surfaces). The obtained abundances profile has been used to compute the line emission of the core as a function of the visual extinction using a Monte Carlo radiative transfer code. Finally, the model predictions have been compared to observations. The best agreement between the model predictions and the observations is obtained for a metal abundance of $3 \times 10^{-9}$ with respect to $\mathrm{H}$ nuclei, but the observations are also consistent with a complete depletion of metals on the grain surfaces (that is, with the cosmic rays as the sole source of ionization). 
the binding energies of these two molecules are almost the same ${ }^{11}$, casting the previous interpretation into doubt. Besides, other laboratory measurements ${ }^{12}$ of the dissociative recombination rate of $\mathrm{N}_{2} \mathrm{H}^{+}$-a key parameter to derive $\mathrm{N}_{2}$ abundance from observations of $\mathrm{N}_{2} \mathrm{H}^{+}$- shows that this reaction, which was previously assumed to form $\mathrm{N}_{2}$, instead mostly forms $\mathrm{NH}$. In view of these recent results, the abundance of $\mathrm{N}_{2}$ in molecular clouds has to be re-investigated.

Using a technique that combines the predictions of chemical network ${ }^{13}$ with a radiative transfer model, we have re-analysed the $\mathrm{N}_{2} \mathrm{H}^{+}$emission maps in the $\mathrm{B} 68$ pre-stellar core. This core is an ideal laboratory to study interstellar chemistry, because its physical structure (density and temperature) is well known. The density profile of this core has been inferred from the extinction of background starlight ${ }^{14}$, and is found to be well described by the equations for a pressureconfined, self-gravitating isothermal sphere that is critically stable. In addition, the gas temperature inside the core has been constrained by comparison of a chemical/physical model to observations of $\mathrm{C}^{18} \mathrm{O}$ and ${ }^{13} \mathrm{CO}$ line emission ${ }^{15}$.

To model the $\mathrm{N}_{2} \mathrm{H}^{+}$emission, it is crucial to constrain the abundance of electrons and $\mathrm{CO}$, which set the destruction rate of this ion. The CO abundance has been determined in the core by modelling and observations of transitions of $\mathrm{CO}$ isotopologues ${ }^{15}$. A ready source of ionization is the ultraviolet photons emitted by nearby stars, but these cannot penetrate the interior of dense molecular cores. Consequently, cosmic rays are believed to provide the main source of ionization. In addition, pre-existing gaseous refractory metals of low ionization potential (for example, $\mathrm{Fe}$ and $\mathrm{Mg}$ ) can contribute to the electron abundance because they can exchange charge with molecular ions, producing $\mathrm{Fe}^{+}$and $\mathrm{Mg}^{+}$, which are destroyed relatively slowly by radiative recombination ${ }^{16}$. In our model, we have assumed a standard value for the cosmic ionization rate $\left(\zeta=3 \times 10^{-17} \mathrm{~s}^{-1}\right)$ and we have determined the electron abundance from observations of $\mathrm{H}^{13} \mathrm{CO}^{+}(1-0)$ line emission (see Fig. 1). Figure 2 shows the predicted electron abundance structure in the core. Our estimates are in agreement with electron abundances obtained in other cores ${ }^{16,17}$.

The electron and $\mathrm{CO}$ abundances determine the destruction rate of $\mathrm{N}_{2} \mathrm{H}^{+}$. The cosmic ionization rate sets the $\mathrm{H}_{3}^{+}$abundance, and thus the $\mathrm{N}_{2} \mathrm{H}^{+}$production rate from $\mathrm{N}_{2}$. With these two factors constrained, the $\mathrm{N}_{2} \mathrm{H}^{+}$abundance depends solely on the $\mathrm{N}_{2}$ abundance inside the core (see Supplementary Information). Assuming that all nitrogen is initially in the form of $\mathrm{N}_{2}$, we found that the predicted $\mathrm{N}_{2} \mathrm{H}^{+}(1-0)$ line fluxes are consistently higher than the

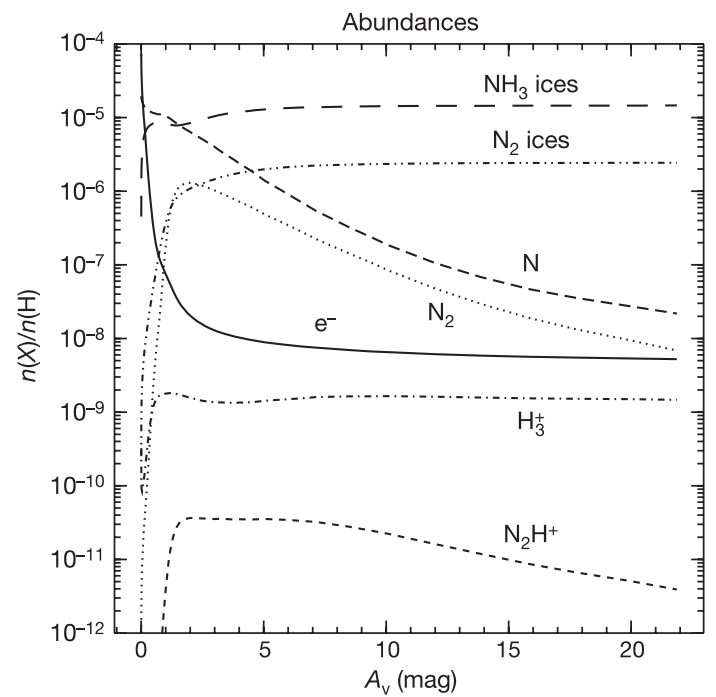

Figure 2 | Abundances of electrons and nitrogen-bearing species inside the core, at $t=10^{5} \mathrm{yr}$. observed ones (see Fig. 3). Therefore, we suggest that the nitrogen element in cold molecular clouds is mostly atomic and not molecular, as previously thought. These observations require only a quarter of the elemental nitrogen to be initially in the form of N. Indeed, earlier $\mathrm{N}_{2} \mathrm{H}^{+}$observations did seem to support this idea ${ }^{18}$.

This result has important implications on our understanding of the composition of comets. Comets are thought to be, in whole or in part, aggregates of interstellar grains that survived the formation of the solar nebula ${ }^{5}$. The striking similarities between the composition of cometary and interstellar ices ${ }^{19}$ favour this hypothesis. However, some differences exist: the total amount of nitrogen is known to be deficient in comets, with respect to the solar value $e^{20}$. This deficiency has been linked to the low abundance of $\mathrm{N}_{2}$ in cometary ices ${ }^{2,3}$. This low abundance is puzzling, if nitrogen is mostly in the form of $\mathrm{N}_{2}$ in cold dark cloud as has long been assumed. However, it has previously been suggested that the low $\mathrm{N}_{2}$ abundance in comets could be a result of a high $\mathrm{N} / \mathrm{N}_{2}$ ratio $^{6}$. Indeed, our work indicates that only a small fraction of nitrogen is in form of $\mathrm{N}_{2}$ in the gas phase (see Fig. 2). Therefore, the amount of $\mathrm{N}_{2}$ that freezes on the grain surfaces is also small with respect to the total nitrogen abundance. Our model predicts that the $\mathrm{N}_{2}$ abundance in grain mantles with respect to water will be only about $1 \%$. Thus the lack of molecular nitrogen in cometary nuclei can now be understood.

The overall nitrogen deficiency in comets is more difficult to understand. In principle, if nitrogen is mostly in atomic form in the gas phase, only a fraction of the total nitrogen would be incorporated in grain mantles, because atomic nitrogen has a very low binding

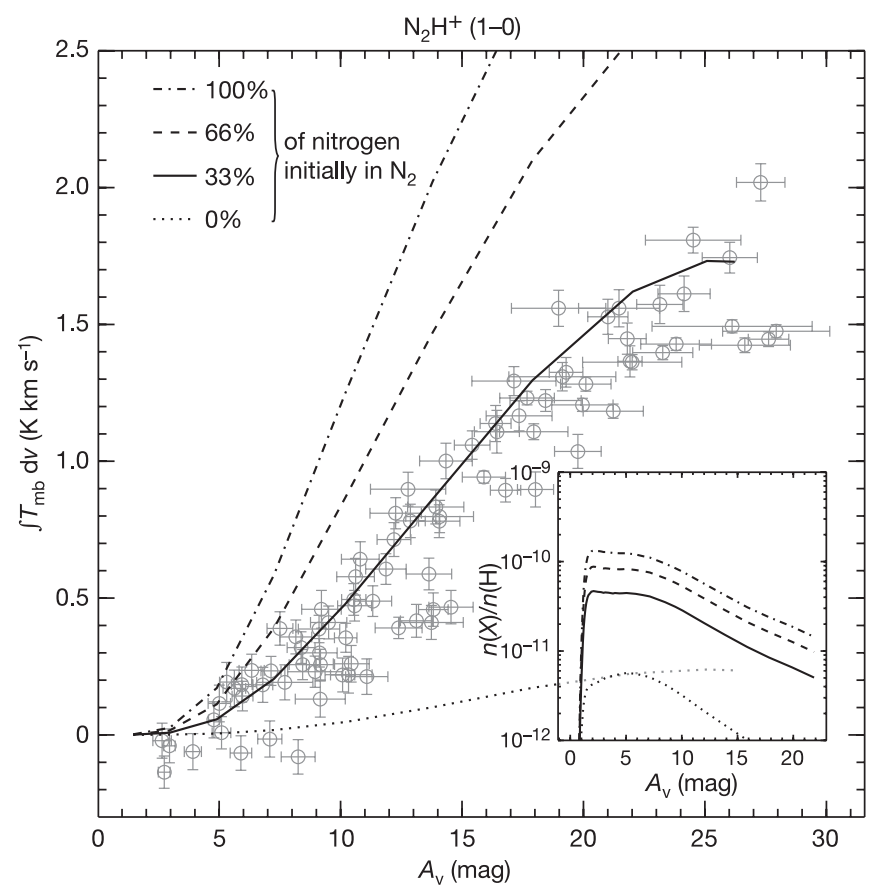

Figure 3 Determination of the fraction of the elemental nitrogen in molecular form. Grey points with error bars $(1 \sigma)$ represent the observed $\mathrm{N}_{2} \mathrm{H}^{+}(1-0)$ line intensities as a function of $A_{\mathrm{v}}$ (ref. 9). The black lines represent the model predictions for different fractions of the nitrogen element initially in the form of $\mathrm{N}_{2}$. We refer to initial abundances as the abundances at $t=0$ in our time-dependent chemical modelling (see Supplementary Information for further details). We have adopted an elemental nitrogen abundance of $2 \times 10^{-5}$ with respect to $\mathrm{H}_{\text {. }} \mathrm{N}_{2} \mathrm{H}^{+}$has an hyperfine structure owing to the interaction of the molecular electric field gradient with the electric quadrupole moments of the two nitrogen nuclei ${ }^{24}$. This interaction splits the 1-0 line into seven components that must be modelled properly to obtain a correct opacity for the line emission. We have used a recent technique to handle the hyperfine structure within a Monte Carlo radiative transfer code ${ }^{25}$, and properly computed the emission. 
energy, and therefore does not condense on the grain surface. However, our model and observations require atomic nitrogen to form ammonia on grain mantles by grain surface reaction, in order to prevent it returning to the gas phase and forming $\mathrm{N}_{2}$. In the B68 prestellar core, our model indicates that the main nitrogen reservoir is ammonia ice, with an abundance of about $7 \%$ with respect to water. Although this value is compatible with ammonia ice abundances measured in dark clouds ${ }^{21}$, it is much higher than the ammonia abundances observed in comets $(\sim 1 \%)^{3}$. Our work could be reconciled with the nitrogen deficiency in comets if the atomic oxygen in the gas phase were lower than the one predicted by our model. This would prevent the formation of $\mathrm{N}_{2}$ through NO, providing more atomic nitrogen in the gas phase and less $\mathrm{NH}_{3}$ (see Supplementary Information).

This result could also explain the nitrogen isotopes anomalies $\left({ }^{15} \mathrm{~N} /{ }^{14} \mathrm{~N}\right)$ observed in some meteorites and interplanetary dust particles ${ }^{4} .{ }^{15} \mathrm{~N}$ enrichments are probably the result of low-temperature chemistry, although dark cloud chemical models predict only modest enhancements of the ${ }^{15} \mathrm{~N} /{ }^{14} \mathrm{~N}$ ratio ${ }^{22}$. However, this fractionation is greatly increased if a large portion of the nitrogen is in atomic form ${ }^{6}$.

Finally, this work complements the recent detection of the $\mathrm{N}_{2}$ in a diffuse molecular cloud ${ }^{23}$. The $\mathrm{N}_{2}$ abundance is expected to be much lower than in dense molecular clouds, because strong ultraviolet fields in diffuse molecular clouds photo-dissociate $\mathrm{N}_{2}$. The abundance measured in a diffuse molecular cloud $\left(1.6 \times 10^{-7}\right.$ with respect to $\mathrm{H}$ nuclei) is about an order of magnitude lower than the one estimated in the present work $\left(1.1 \times 10^{-6}\right.$, see Fig. 2$)$, and is therefore consistent with this picture.

In conclusion, this study emphasizes the similarity between the compositions of grain ices and of cometary nuclei, meteorites, and interplanetary dust particles. In situ analysis of cometary nuclei will be needed to confirm our hypothesis. The ESA's Rosetta missionwhich will land on the comet 67P/Churyumov-Gerasimenko-will provide new insights into the nucleus composition of the comet, and any potential link to interstellar chemistry.

\section{Received 27 February; accepted 16 May 2006.}

1. Herbst, E. \& Klemperer, W. The formation and depletion of molecules in dense interstellar clouds. Astrophys. J. 185, 505-534 (1973)

2. Cochran, A. L., Cochran, W. D. \& Barker, E. S. N ${ }_{2}^{+}$and $\mathrm{CO}^{+}$in Comets $122 \mathrm{P} / 1995$ S1 (deVico) and C/1995 O1 (Hale-Bopp). Icarus 146, 583-593 (2000).

3. Iro, N., Gautier, D., Hersant, F., Bockelée-Morvan, D. \& Lunine, J. I. An interpretation of the nitrogen deficiency in comets. Icarus 161, 511-532 (2003).

4. Messenger, S., Keller, L. P., Thomas, K. L. \& Walker, R. M. Nitrogen petrography in two ${ }^{15} \mathrm{~N}$-rich IDPs. Meteorit. Planet. Sci. 31, A88 (1996).

5. Irvine, W. M., Schloerb, F. P., Crovisier, J., Fegley, B. \& Mumma, M. J. Comets: a link between interstellar and nebular chemistry. Protostars Planets IV, 1159-1200 (2000)

6. Charnley, S. B. \& Rodgers, S. D. The end of interstellar chemistry as the origin of nitrogen in comets and meteorites. Astrophys. J. Lett. 569, L133-L137 (2002)
7. Linke, R. A., Langer, W. D. \& Guelin, M. Detection of $\mathrm{H}^{15} \mathrm{NN}^{+}$and $\mathrm{HN}^{15} \mathrm{~N}^{+}$in interstellar clouds. Astrophys. J. Lett. 271, L85-L88 (1983).

8. Tafalla, M., Myers, P. C., Caselli, P., Walmsley, C. M. \& Comito, C. Systematic molecular differentiation in starless cores. Astrophys. J. 569 815-835 (2002).

9. Bergin, E. A., Alves, J., Huard, T. \& Lada, C. J. $\mathrm{N}_{2} \mathrm{H}^{+}$and $\mathrm{C}^{18} \mathrm{O}$ depletion in a cold dark cloud. Astrophys. J. Lett. 570, L101-L104 (2002).

10. Pagani, L., Pardo, J.-R., Apponi, A. J., Bacmann, A. \& Cabrit, S. L183 (L134N) revisited. III. The gas depletion. Astron. Astrophys. 429, 181-192 (2005).

11. Öberg, K. I. et al. Competition between $\mathrm{CO}$ and $\mathrm{N}_{2}$ desorption from interstellar ices. Astrophys. J. Lett. 621, L33-L36 (2005).

12. Geppert, W. D. et al. Dissociative recombination of $\mathrm{N}_{2} \mathrm{H}^{+}$: Evidence for fracture of the NN bond. Astrophys. J. 609, 459-464 (2004).

13. Bergin, E. A., Langer, W. D. \& Goldsmith, P. F. Gas-phase chemistry in dense interstellar clouds including grain surface molecular depletion and desorption. Astrophys. J. 441, 222-243 (1995).

14. Alves, J. F., Lada, C. J. \& Lada, E. A. Internal structure of a cold dark molecular cloud inferred from the extinction of background starlight. Nature 409, 159-161 (2001).

15. Bergin, E. A. et al. The thermal structure of gas in pre-stellar cores: a case study of Barnard 68. Astrophys. J. (in the press).

16. Caselli, P., Walmsley, C. M., Terzieva, R. \& Herbst, E. The ionization fraction in dense cloud cores. Astrophys. J. 499, 234-249 (1998)

17. Caselli, P. et al. Molecular ions in L1544. II. The ionization degree. Astrophys. J. 565, 344-358 (2002).

18. Womack, M., Ziurys, L. M. \& Wyckoff, S. Estimates of $\mathrm{N}_{2}$ abundances in dense molecular clouds. Astrophys. J. 393, 188-192 (1992).

19. Bockelée-Morvan, D. et al. New molecules found in comet C/1995 O1 (Hale-Bopp). Investigating the link between cometary and interstellar material. Astron. Astrophys. 353, 1101-1114 (2000).

20. Wyckoff, S., Tegler, S. C. \& Engel, L. Nitrogen abundance in comet Halley. Astrophys. J. 367, 641-648 (1991).

21. Gibb, E. L., Whittet, D. C. B., Boogert, A. C. A. \& Tielens, A. G. G. M. Interstellar ice: the Infrared Space Observatory legacy. Astrophys. J. Suppl. 151, 35-73 (2004).

22. Terzieva, R. \& Herbst, E. The possibility of nitrogen isotopic fractionation in interstellar clouds. Mon. Not. R. Astron. Soc. 317, 563-568 (2000).

23. Knauth, D. C., Andersson, B.-G., McCandliss, S. R. \& Warren Moos, H. The interstellar $\mathrm{N}_{2}$ abundance towards HD 124314 from far-ultraviolet observations. Nature 429, 636-638 (2004).

24. Caselli, P., Myers, P. C. \& Thaddeus, P. Radio-astronomical spectroscopy of the hyperfine structure of $\mathrm{N}_{2} \mathrm{H}^{+}$. Astrophys. J. Lett. 455, L77-L80 (1995).

25. Keto, E., Rybicki, G. B., Bergin, E. A. \& Plume, R. Radiative transfer and starless cores. Astrophys. J. 613, 355-373 (2004).

Supplementary Information is linked to the online version of this paper at www.nature.com/nature.

Acknowledgements This work is supported by the National Science Foundation. S.M. is grateful to E. Herbst for discussions about the $\mathrm{N}_{2}$ formation reaction rates. S.M. also thanks E. Keto for discussions about the radiative transfer of $\mathrm{N}_{2} \mathrm{H}^{+}$hyperfine structure lines.

Author contributions S.M. and E.A.B. performed the chemical and radiative transfer modelling presented in this paper. S.M. wrote the paper. All authors discussed the results and commented on the manuscript.

Author Information Reprints and permissions information is available at npg.nature.com/reprintsandpermissions. The authors declare that they have no competing financial interests. Correspondence and requests for materials should be addressed to S.M. (smaret@umich.edu). 